\title{
Levantamento bibliográfico sobre tecnologias assistivas baseadas em realidade aumentada para desenvolvimento de atividades com crianças autistas.
}

\author{
Jonas C. Boechat ${ }^{1}$, Fernando M. Magalhães ${ }^{2}$, Otávio M. Dantas ${ }^{1}$, Eunice P. dos Santos \\ Nunes $^{3}$, Elmo B. de Faria ${ }^{3}$, Luciana C.L. de Faria Borges ${ }^{3}$ \\ ${ }^{1}$ Faculdade de Engenharia de Várzea Grande - Univers. Federal de Mato Grosso(UFMT) \\ ${ }^{2}$ Departamento de Engenharia Elétrica - Univers. Federal de Mato Grosso (UFMT) \\ ${ }^{3}$ Instituto de Computação - Univers. Federal de Mato Grosso (UFMT) \\ jonas_boechat@hotmailcom, fernandinho_ferinha@hotmail.com, \\ otavio-dantaselive.com, eunicedufmt.br, elmodic.ufmt.br, \\ lucianafariaborges@gmail.com
}

Resumo. Este trabalho é resultado de um levantamento bibliográfico feito a partir de pesquisas a respeito de uma Tecnologia Assistiva baseada em realidade aumentada para o desenvolvimento no tratamento de crianças autistas. A pesquisa dos artigos foram feitas a partir de uma busca com strings no acervo do IEEE contendo limitações quanto a data de publicação e coerência com o tema proposto. Os resultados coletados mostram que a realidade aumentada, e outras ferramentas tecnológicas, corroboram de forma conveniente para para o desenvolvimento da criança.

\section{Introdução}

As escolas do Brasil vêm apresentando um aumento significativo de alunos com deficiência. Dentre esses alunos, é possível destacar uma grande incidência de alunos com transtorno do espectro autista (TEA).(INEP, 2018)

O TEA é um transtorno do neurodesenvolvimento de crianças que possuem como principais características as dificuldades na interação social, comunicação, comportamentos repetitivos e interesses restritos, apresentando também algumas sensibilidades sensoriais, dependendo do caso (Rosa, Silva e Aymone, 2018).

Crianças com este tipo de deficiência costumam aprender mais devagar que crianças que não apresentam tais limitações e, por isso, precisam de mais assistência técnica.(Lin, Chao e Wei, 2010)

Atualmente, o avanço da tecnologia contribui cada vez mais para a inclusão de alunos no ensino, em especial aqueles com deficiência. Conforme Galvão Filho (2009), Tecnologias Assistivas são recursos e serviços que visam facilitar o desenvolvimento de atividades da vida diária por pessoas com deficiência, no sentido de aumentar capacidades funcionais e assim promover a autonomia e independência de quem as utiliza. Logo, desenvolver 
Tecnologias Assistivas promove não só diferentes meios de aprendizagem, mas também qualidade de vida as pessoas que possuem este tipo de deficiência.

Dessa forma, o desenvolvimento de Tecnologias Assistivas para promoção de atividades com crianças com TEA permitem, por exemplo, que as mesmas aprimorem aspectos básicos como lateralidade, comunicação, habilidades e outros aspectos. Sendo assim, crianças com TEA podem usufruir destas tecnologias para o desenvolvimento de atividades, já que as mesmas possuem dificuldade de comunicação por deficiência no domínio da linguagem e no uso da imaginação para lidar com jogos simbólicos, dificuldade de socialização e padrão de comportamento restritivo e repetitivo.

O uso da Realidade Aumentada (RA) cada vez mais se faz presente no desenvolvimento de Tecnologias Assistivas. De acordo com Lin, Chao e Wei (2010), a RA é apresentada como uma excelente interface de usuário para aplicativos de computação onipresentes que são recursos utilizados em qualquer lugar e momento, porque permite a navegação intuitiva e de informações referenciadas por local. A interação com essas entidades ocorre em tempo real, fornecendo feedback convincente ao usuário e dando a impressão de interação natural.

Levando-se em conta as especificidades das crianças com TEA e o potencial das tecnologias baseadas em RA que recursos para desenvolver atividades que possam promover técnicas de inclusão e também a evolução de fatores intelectuais e motores.

Dessa forma, este artigo contribui na análise da literatura bibliográfica atual, de forma a levantar pesquisas que apresentam investigações sobre Tecnologias Assistivas baseadas em RA para auxiliar o desenvolvimento de atividades com crianças autistas. Nessa busca, a questão principal de pesquisa é encontrar "De que modo e em quais atividades é possível e mais efetivo o uso do RA para desenvolver Tecnologias Assistivas que contribuam para a promoção de atividades com crianças autistas".

Nas seções a seguir é são mostrados os materiais e métodos da pesquisa, o seu embasamento literário, e por fim os resultados obtidos e o que se pode concluir com o que foi feito.

\section{Materiais e Métodos}

Para atingir tais objetivos, primordialmente foram realizadas buscas de artigos que abordassem Tecnologias Assistivas baseadas em RA para auxiliar atividades com crianças com TEA. Os principais mecanismos de busca usados pelos pesquisadores foram IEEE, ACM, SciELO, Google Acadêmico e alguns sites diversos que possuíam reportagens relacionadas ao tema. A strings de busca utilizadas nas referidas bases de dados foram "augmented reality" AND "autism".

Destas strings, foram obtidos vários resultados e após criteriosa seleção por meio de leitura dos abstracts e leitura completa dos artigos atingiu-se um total de 26 artigos, dos quais foram selecionados apenas 6. Essa seleção foi gerada por meio de critérios de exclusão definidos pela equipe, selecionando trabalhos entre 2014 e 2019 e relacionado ao objetivo primordial do trabalho. Não somente artigos foram utilizados mas também sites que apresentaram dados que seriam de proveitosos ao tema buscado. 
Esses artigos deram a base para responder à questão de pesquisa: "De que modo e em quais atividades é possível e mais efetivo o uso do RA para desenvolver Tecnologias Assistivas que contribuam para a promoção de atividades com crianças autistas".

Os resultados encontrados foram organizados em tabela, apresentando os principais conteúdos dentro da área pesquisada que eles abordam e que embasam esta pesquisa.

\section{Embasamento Literário}

Os artigos selecionados trazem resultados acerca de tecnologias baseadas em RA e que auxiliam a inclusão de crianças com TEA no meio social, atendendo dessa forma, o embasamento desta pesquisa.

Rosa, Silva e Aymone (2018) pesquisaram a melhoria de pranchas de comunicação com os recursos de RA, oferecendo um novo design para auxiliar a comunicação de crianças com TEA. As ferramentas 3D STUDIO MAX e o aplicativo gratuito AURASMA foram utilizados respectivamente, para o desenvolvimento de objetos $3 \mathrm{D}$ e, para desenvolver ações em RA de maneira simplificada, transformando imagens em multimídias reais. A relevância dessa oferta apoia a necessidade apontada por esse trabalho, que mostra a importância de expor e insistir continuamente no processo de aquisição da linguagem de crianças com TEA.

No segundo trabalho encontrado, a falta de imaginação foi identificada como um dos principais sintomas que constituem a tríade de características da condição do espectro do autismo, juntamente com interação social e comunicação. A partir disso, Bai, Blackwell e Coulouris (2015), desenvolveram um sistema interativo que explorou o potencial da RA para conceitualizar visualmente um ambiente de jogo aberto voltado para crianças com TEA e para explorar a imaginação dessas crianças.

Reconhecendo que a RA permitem que as pessoas entendam melhor seus arredores, combinando realidade com conteúdo virtual de maneiras significativas, o sistema foi projetado por meio de uma câmera de vídeo direcionada às crianças com TEA, para que pudessem se visualizar em um monitor adequado para RA, os objetos do mundo real como por exemplo, blocos de madeira que se transformam em carros ou casas. Os resultados foram positivos, com aumento na frequência e a duração das brincadeiras, se comparadas a um sistema não computacional. Vale ressaltar que as diferenças individuais entre os participantes preveem que a eficácia seja gradual para crianças em diferentes condições autistas.

O terceiro trabalho encontrado, visa auxiliar crianças com TEA no campo de comunicação, sendo assim, Nubia et al. (2015), desenvolveram com a utilização de RA uma nova perspectiva para terapia de crianças da clínica Neurohabilitar. A clínica utiliza cartões com figuras de animais, frutas e meios de transporte esperando a reação da criança ao visualizar e reconhecer. A equipe transformou as imagens presentes nos cartões em figuras 3D onde adicionaram sons que poderiam ajudar as crianças no reconhecimento.

O estudo foi feito com 6 crianças de 3 à 9 anos de idade, observando a reação delas com a terapia tradicional e comparando com a terapia utilizando o recurso de RA. Uma melhora significativa foi observada principalmente nos cartões com animais em 3D onde as crianças 
conseguiram com a ajuda dos sons reconhecer o animal presente no cartão. $\mathrm{O}$ estudo foi concluído com uma melhora de $14 \%$ na atenção das crianças presentes.

No quarto trabalho, Silva et al. (2017) apresentam um jogo sério chamado Knowledgemon Hunters, desenvolvido para amenizar a confinação de crianças autistas e auxiliar em seu aprendizado. No jogo foi possível capturar monstros, andando em qualquer área por meio da geolocalização. Ao capturar os monstros a criança acessa objetos de aprendizagem relacionados aos monstros. A RA, neste caso, foi utilizada para gerar interesse na criança, que ao visualizar os monstros no celular e aprendem com ele.

No quinto trabalho, os autores Escobedo et al. (2014) exploram como a RA pode ajudar a redirecionar a atenção de crianças com autismo. Para isso utilizaram um sistema de identificação de objetos móveis (Mobis), um aplicativo móvel de RA que foi desenvolvido para permitir que os professores sobreponham o conteúdo digital sobre objetos físicos. Com a utilização do aplicativo o tempo de permanência dos alunos nas tarefas aumentou em $20 \%$. Os alunos frequentaram as sessões de terapias mais animados ao usá-lo "em movimento" para descobrir objetos no ambiente.

No sexto trabalho, Paiva et al.(2017) realizaram um acompanhamento psicopedagógico semanal, em que as crianças eram submetidas a sessões para medir aspectos relacionados à cognição. O uso da tecnologia de RA neste caso, teve como finalidade, melhorar a interação face a face entre a criança com TEA e psicopedagogo que a acompanha. Portanto, construíram um ambiente de RA, tendo como base os óculos de realidade virtual Google Cardboard.

Com a utilização de um filtro a imagem capturada pela câmera do celular é transformada em um desenho animado, similar a um gibi. Como resultado da suavização do processamento facial através da tecnologia proposta demonstra-se um melhor entendimento de atividades que envolvam as funções executivas.

\section{Resultados Obtidos}

Com este levantamento bibliográfico notou-se a complexidade de trabalhar com crianças com TEA. O estudo aborda a RA como forma de inclusão e desenvolvimento social, sobrepondo o mundo físico com o universo digital, auxiliando os tratamentos e a inclusão social dos usuários. Cada usuário pode reagir de forma diferente às tecnologias baseadas em RA, não se isentando o fato de que em alguns casos esta tecnologia apresenta resultados não compatíveis com o esperado, tornando o processo de desenvolvimento mais específicos para cada caso. A Tabela 1 elenca de forma sintetizada os resultados levantados nessa pesquisa.

Ressalta-se que, foram encontrados trabalhos para melhorar a cognição, comunicação, imaginação, atenção de crianças com TEA. Em todos os trabalhos selecionados a RA mostrou-se um recurso indicado para melhorar a atenção das crianças, auxiliando os métodos tradicionais já utilizados e os tratamentos atuais. 


\section{Tabela 1 . Resultados obtidos pelo levantamento biblíografico}

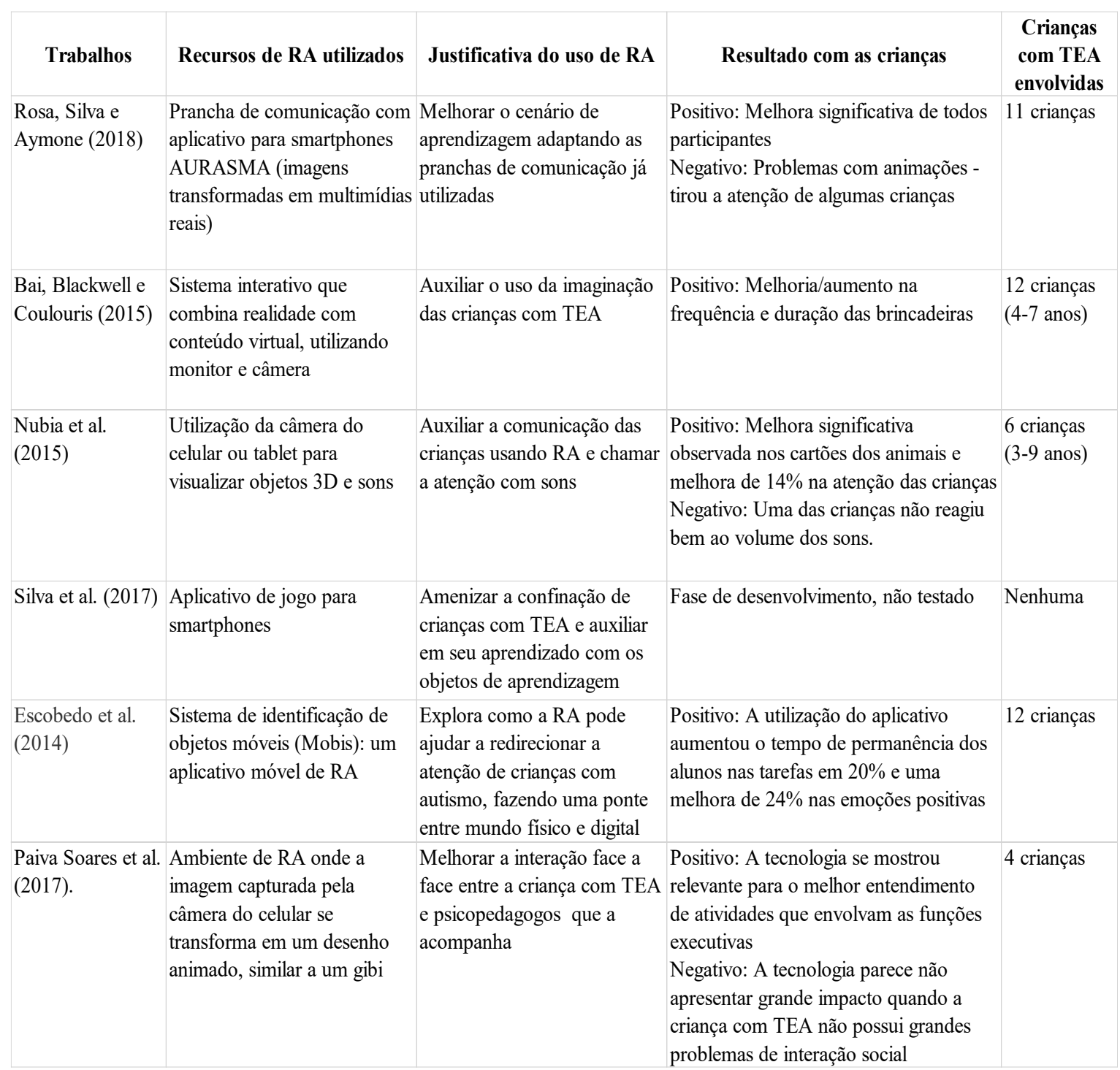

\section{Considerações Finais}

O objetivo da pesquisa foi alcançado, uma vez que foram encontrados trabalhos que responderam à questão desta pesquisa. Os resultados indicaram que tecnologias assistivas 
baseadas em RA podem ser uma ferramenta de apoio à crianças com autismo, sendo recomendável pesquisas que ampliem essas possibilidades.

Ao longo deste trabalho encontrou-se a dificuldade em obter um número considerável de pesquisas relacionadas ao nosso foco e referentes ao período de busca entre o ano atual e 2017, o que nos forçou à ampliação desse período até o ano de 2014.

Assim, um dos trabalhos futuros poderia ser o fomento de novas investigações atendendo o tema deste trabalho não apenas para crianças com TEA, mas com outras deficiências.

\section{Referências}

Instituto Nacional de Estudos e Pesquisas Educacionais (INEP). Censo Escolar. (2018). Brasília: MEC, 2018.

Lin, C. Y., Chao, J. T., \& Wei, H. S. (2010). Augmented reality-based assistive technology for handicapped children. 3CA 2010 - 2010 International Symposium on Computer, Communication, Control and Automation, 1, 61-64.

Galvão Filho, T. A. (2009). Tecnologia assistiva para uma escola inclusiva: apropriação, demanda e perspectivas.

Rosa, V. I.; Silva, R. P.; Aymone, J. L. F.(2018). Design inclusivo: processo de desenvolvimento de prancha de comunicação alternativa e aumentativa para crianças com transtorno do espectro do autismo utilizando realidade aumentada: ,. Design e Tecnologia, v. 8, n. 15, p. 51-67.

Bai, Z., Blackwell, A. F., \& Coulouris, G. (2015). Using Augmented Reality to Elicit Pretend Play for Children with Autism. IEEE Transactions on Visualization and Computer Graphics, 21(5), 598-610.

Nubia, R. M. et al. (2015). Development of a mobile application in augmented reality to improve the communication field of autistic children at a Neurorehabilitar Clinic. 2015 Workshop on Engineering Applications - International Congress on Engineering (WEA).

Silva, S. D. et al. (2017). Knowledgemon Hunter: A Serious Game with Geolocation to Support Learning of Children with Autism and Learning Difficulties. 2017 19th Symposium on Virtual and Augmented Reality (SVR).

Escobedo, L. et al. (2014). Using Augmented Reality to Help Children with Autism Stay Focused. IEEE Pervasive Computing, 13(1), 38-46.

Paiva Soares, K. et al. (2017). Preliminary Studies With Augmented Reality Tool To Help In Psycho-pedagogical Tasks With Children Belonging To Autism Spectrum Disorder. IEEE Latin America Transactions, 15(10), 2017-2023. 